\title{
Vegetable Oil Paints
}

\author{
L. L. CARRICK, University of Michigan, \\ Ann Arbor, Michigan
}

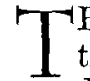
HE Egyptians recognized the medicinal properties of linseed oil but were unacquainted with its drying properties. Ludius used oil in encaustic paints during the reign of Agustus (63 B.C.-14 A.D.). Vitruvius, during the same period, prepared a varnish from Punic wax and a little oil. Dioscorides (69-30 B.C.) recognized the dispersion properties of oils for natural resins. Pliny the author (A.D. 23-70) makes no mention of the use of oil by artists or painters. Lucanus (A.D. 37-68) mentioned the use of oil to improve the working characteristics of color for paints. Galen (A.D. 131-200) coagulated drying oils, such as hempseed, nut, and linseed oils by means of litharge, white lead, and umber. Actius (A.D. 540) emphasized to the arts the drying properties and varnish potentialities of linseed, castor, and nut oil used by Gilders and encaustic painters. Lucea (A.D. 800) was the first to record instruetions for preparing varnish from resin and boiling linseed oit. In the 11th century Theophilis, a monk, gave explicit instructions for melting resin until clear, adding hot linseed, olive, walnut, or poppyseed oils and continuing to heat the mixture until a drop remained clear upon cooling. He complained about the slow drying characteristics of the film and resorted to exposure to the sun to accelerate drying. In principle this is the same method employed at the present time in many varnish cooks.

Eraclius and Petrus (12th to 13th centuries A.D.) recorded methods for the extraction and refining of oil by adding small quantities of lime, followed by heating and continued skinning. Petrus also used white lead as a drier and bleached oils in the sun with frequent agitation. The protective value of drying oils was generally accepted in France, England, and Germany in the 13th and 14th centuries. Jacobus de Tholeto, 1450, was among the first to suggest that metallic salts be cooked with the resin and oil to hasten the subsequent drying rate. No mention of thinning varnish was made until 1736 , when Alberti of Magdeburg thinned varnish with turpentine, which remained the principal thinner of varnish until 1880 after which petroleum distillate was commercially employed. Watin, 1773 , realized the commercial possibilities of varnish and published a book in which he offered varnish for sale to other craftsmen so that they would not have to cook their own varnish. In the 13 th and 14 th centuries the records show that Lübeck, Kolmar, van Eyck, Cennini, Alcherius, and other artists and craftsmen demonstrated the value of common oils, such as raw and refined walnut, linseed, hempseed and poppy seed, for use as vehicles in portrait and decorative paints. At Canterbury (13th century) and Ely (14th century) the records disclose that oil was used in decorative paints. Oil was first used commercially in the manufacture of varnish in England in 1790, France 1820, New York 1828, Germany 1830, and Austria 1843.

The date of the first use of oil in paints has been lost in obscurity. The earliest paints were little more than colored rock or clays dispersed in plant juice, milk, egg white, natural glues, animal and/or vege- table waxes, pitches, or the oils from wild nuts or seeds. The early use of raw walnut, linseed, poppy seed, olive, or hempseed oils has been noted together with the advent of their refined and bleached modification. Chinese tung oil, according to D'Incarville, 1735, was first a common adulterant of Chinese lacquers and did not commercially replace linseed oil in many varnishes in the United States until 1900. Enamels were unknown before the beginning of the present century. Prior to this time it was common practice to apply a flat oil paint of the desired color and follow it with a coat of clear varnish to obtain the needed gloss. About this time it was found that pigments could be ground into a varnish vehicle and give a product which was stable in the container. This product would give in one operation a film of the desired color which had high gloss and good levelling.

The commercial use of perilla, fish, soybean, oiticica, sunflower, safflower, and dehydrated castor oil in paints are attributed to developments in the 20th century.

Such paints or metal primers, sanding undercoats, baking colored enamels, and oil stains are among recent developments required by the industrial advances of a mechanical age and the tremendous increased demand as a result of shortened production schedules, production lines, and increased market pressure.

Although modern scientific studies have uncovered and developed many substitutes and partial replacements for drying oils, it has not been able to decrease their importance to any appreciable extent. Today's paint and varnish industry and many synthetic resin industries would be greatly handicapped if forced to formulate the variety of modern organic protective coatings industrially, without the aid of the oil products of nature.

There are a large number of factors which have great influence upon the utility of organic protective coating oils, any one of which may spell the difference between usability or be completely or largely responsible for the oil's unusability. These factors may be roughly grouped into two categories, technical factors and economic factors.

The economic factors, in turn, are dependent upon such subfactors as steady availability of the oil, stability of the oil supply, physical and chemical stability of the oil, low price of the oil, and stability of the price. The technical factors may be further divided into the properties of the liquid oil and the properties of the drying or dried oil film. A few of the liquid oil properties which must be evaluated in terms of the formulation requirements are acidity; color; odor; viscosity; amount of saturated fatty acids; amount of unsaturated fatty acids; degree and arrangement of the unsaturated double bonds in the fatty acid; drying rate and time; solubility in common solvents; miscibility with other oils; skinning time, stability in storage; stability under heat, flow, leveling, break, and wetting characteristics. 


\section{Purpose}

It is the purpose of this paper to point out the oils that are used in today's organic finishes. We will confine most of our discussion to the consideration of the direct applications to paints and not unduly emphasize the important and widespread uses of oils in the manufacture of varnish and synthetic resin, except where their products are used in paints. We shall briefly call attention to the composition of the oils so as better to appreciate the uses of oil whether it is raw, refined, treated, modified, improved, or synthetic.

In the film the dried oil properties such as hardness, flexibility, elasticity, resilience, color, color retention, permeability to fluids, waterproofness, adhesion, solubility, resistance to ultraviolet light, resistance to acids and alkalies, gloss retention, bleeding, resistance to heat and abrasion, transparency, coefficient of expansion for conditions under which used, and other properties for specific application must be met if the oil is to meet with wide-spread favor. The continued use of oil in a paint vehicle without doubt must reflect the fact that the above mentioned economic and technical factors have in a large measure been met. With few exceptions science has found other materials than oil, which excel in one or more of the liquid or film properties, but these competitive materials are deficient in other characteristics, and, in addition, lack the ability to meet the overall desirable and well-rounded characteristics of natural or processed oil. Not all oils meet all of these properties, some are superior in certain properties but less suitable in others. At this point it is well to point out that the selection of an oil for a particular formulation is not generally based upon a single factor but is a combination of both the technical and economic factors. In some instances the economic factor is paramount. Economically in some instances, it is desirable to use a high-priced treated oil while in others a lower priced oil as soybean or tall oil may be entirely satisfactory. A delicate point for the manufacturer to decide at this point is what to offer in competitive bidding on a performance specification request.

Since it is the function of this paper primarily to present the uses of the oils in paints, sufficient theory has been presented by previous speakers to direct attention to the structure of the oil molecule and the possible reactions of the oil in drying that we may now mention other characteristics of oils:

1. All natural drying oils contain variable amounts of saturated fatty acid chains which do not dry but are important considerations in plasticizers to decrease hard setting.

2. All natural drying oils contain unsaturated double bonds.

3. All natural drying oils are esters, thus are capable of being saponified, and as a consequence they are not very alkaliresistant. Important in polymerization and drying and will help in determining type of finishes which may be formulated with them.

4. Most natural drying oils contain an insufficient number of unsaturated double bonds. Important in drying, plasticiz ing, and polymerization.

5. Most natural drying oils contain the unsaturated double bonds in unfavorable positions. Important consideration in determining the type and extent of polymerization, processing of oils, and charaeteristics of the drying oil film.

6. The triglyceride structure prevails in natural drying oils and is not the most desirable under all types of applieation of the oil in protective coatings, alkyds, varnish, and synthetic resins.

7. Once a film of oil has dried hard, the reactions within the oil film do not stop but continue and eventually destroy the oil film. Important in all films where unprocessed oils are used and may be important in many processed oils.

8. Sorne films of oil, more than others, show as a result of continued reaction in the oil film, after it sets, a decided tendency to yellow. They are said to have bad color retention. The type of unsaturation and the amount of the unsaturates are important in estimation of the change in color in varnish, lacquers, paints, and enamels, especially white and tints.

9. Oils which are blown with air at $200-400^{\circ} \mathrm{F}$ for several hours show the effect of oxygen attack on the double bonds by an increase in viscosity, deepened color, low solubility in nonpolar solvents, and greater reactivity with basic pigments. Blown oils have remarkable wetting characteristics but are in many properties inferior to kettle bodied oils.

10. Oils which are heat bodied in the absence of air show a gradual increase of viseosity which eventually leads to a solid gel where the oil molecules are united at the double bonds through primary valences without any intermediary oxygen linkage. These oils show improved properties over those of the raw oils. These oils have better flow and leveling characteristics. The films dry harder and faster. The films are glossier and generally have improved resistance to the weather and wear. The bodying of the oil permits the addition of volatile thinner without unduly lowering the viscosity.

11. The raw oils may be used without the addition of undue amounts of volatile thinners.

12. Many raw oils are unusable without kettle treatment or blending.

13. Evidence points to the mixed triglyceride molecular structure of drying oils. A mixed triglyceride structure gives, during film formation, a more homogeneous structure to the film and improved characteristies among which are waterproofness and wear.

14. The general utility of the oil is dependent upon the number of fatty acids which enter into the structure of the mixed triglyceride molecules in any oil. Thus two fatty acids make possible a maximum of six theoretical triglyceride struetures. The number increases rapidly with each additional fatty acid found in an oil and the type of unsaturated system.

Let us consider the oils separately and note their application in paints. Many of the characteristies of the paint film are noted for each type of oil in individual formulations or dried films.

\section{Linseed Oil}

The consumption of linseed oil in the United States is more than that of all other vegetable oils combined. Although linseed oil was not the first vegetable oil to be used by painters and craftsmen who prepared their own paint yet its physical and chemical properties, together with its agricultural potentialities, quantity production, and the prodigious amount of research which has been directed toward its utilization, have made linseed oil the standard of eomparison to evaluate other drying oils.

In $1938,821 / 2 \%$ of all drying oils consumed in the U. S. was linseed oil. Of the amount $61 \%$ was processed in factories, and the balance, $39 \%$, was used mostly by painters in the production of mixed-on-thejob-paints. A breakdown of the $61 \%$ reveals that the paint and varnish factories are $45.5 \%$, the linoleum and oil cloth industry $11.9 \%$, and the printing ink industry $3.6 \%$. Today with the shift from mix-onthe-job-paints to ready-mixed packaged paints the percentage of factory consumed linseed oil has increased to $93 \%$ of the total. A comparison of the factory drying oil consumption for the years 1938 and 1949 indicate the shift from a one- or two-pigment mix-on-the-job-paint with linseed oil as the vehicle to a multiple pigment paint of varied vehicles.

By far the greatest amount of linseed oil is consumed in outside house paints. In this field it has few peers. Its few weaknesses are unimportant. These are offset by its well rounded balance of liquid and film characteristics. 
Raw linseed oil tops the list of all vehicle components in one property, the wetting of ferrous surfaces. It is the most used single vehicle for primer or shop coats on structural steel. On sandblasted, flame brushed, or even the ferrous surfaces where only the very loose mill scale has been removed, the oil impregnates the rust and affords an almost perfect wetting on all these types of surfaces and, upon drying, a most adhesive film.

Raw linseed oil in combination with red lead and zine dust has remarkable adhesion to cleaned and unweathered zinc or galvanized surfaces.

Because of the well rounded properties, universally known characteristies, and availability of raw linseed oil, methods have been developed to treat it, refine it, modify it, improve it, blend it, combine it chemically with other materials, and adapt it to almost every possible protective coating problem. Linseed oil satisfies more of the requirements of the protective coating industry than any other single oil. This is in spite of its somewhat slow drying rate; undesirable yellowing characteristics; its water permeability as compared to some varnishes and synthetics; structural disadvantages like saponifiability; continued oxidation after the original "dry hard" of the film, and poor color retention.

Raw or refined linseed oil blended with $10-15 \%$ soybean oil gives an excellent mixture, which dries to a homogeneous film. The film is more elastic and will not turn brittle under conditions that would effect a film of $100 \%$ pure linseed oil. Such oils may collect a little dirt.

Raw or refined linseed oil may be blended with $50 \%$ or less of dehydrated castor oil to overcome its poor drying characteristics and tackiness. The film of the blend still retains the water-resistance properties of DCO. Raw or refined linseed oil has been blended successfully. with sesame, hempseed, sunflower, safflower, raisin seed, and walnut oils in the preparation of an outside house-paint vehicle. The linseed oil should have a high iodine number, and the percentage of these oils should be about $18-25 \%$ of the oil blend.

\section{Acid Refined Linseed Oils}

Acid refined linseed oil is used as a grinding oil for pigments. The viscosity is usually less than A (G.H.S.). It wets pigments readily and produces paints with the characteristic brushability demanded by painters. It may be almost colorless hence it does not affect the color of white or delicate tints. The low acid numbered oil is especially adapted for grinding zine or titanium pigments. The high acid number 9-16 of acid refined linseed oil is used to produce puffy paints. It reacts during dispersing with white lead, producing lead soaps which serve as the skeleton to prevent rapid settling.

Acid refined oils with acid number, 1.5 , are used as a grinding oil with excellent wetting and levelling properties which are used to form films of high luster. The acid refined oil is suited for grinding zinc oxides and other reactive pigments.

Acid refined oils may be blended with $10 \%$ heavy bodied oil to produce enamel grinding oils, which have excellent brushing qualities, yielding brilliant light colored films suitable for the highest grade of enamels.

\section{Linseed Oil Alkyds}

Linseed oil alkyds are used in under water primers because of the excellent adhesion.

Long oil linseed alkyds are used in exterior house paints, trim paints, and exterior enamels. The use of alkyds provides excellent gloss and color retention and durability under trying conditions. The use of a little heat bodied oil with alkyds in trim paints improves flow, levelling, and brushing qualities.

Linseed alkyds of the proper oil length are used in aluminum paints, heat resisting zinc dust paint, primer paint, grinding and flushing liquids, water resistant paints, fast drying primers and paints, outside house paint, white and colored enamels, automotive primers, maintenance paints, porch and floor paints, traffic paints, railway paints, red lead primers, ink and coatings for silk screens.

Alkyds may carry a blend of linseed and soybean oils to produce vehicles for special primers and enamels for automobile refinishing.

Linseed alkyd finishes with respect to hardness, flexibility, and general resistance to wear are among the finest vehicles used in protective coatings. The alkyd after production retains the stamp of the original oil in appearance, odor, taste, and most of the original oil's physical and chemical properties. Only in drying and bodying do they show noteworthy differences from their parent oil.

\section{Blown Linseed Oil}

Although blown or oxidized linseed oils are inferior in many characteristies to heat bodied oils, yet their outstanding pigment wetting characteristics have made them a desirable addition to poorly-wetting vehicles. They also improve flowing and levelling properties of vehicles at lower cost than by the use of bodied oils. Since they have a viscosity $Y$ to $Z_{3}$, they must be cut with sufficient thinner to reduce the viscosity to the desired workable viscosity. Blown castor oil added to blown linseed oil improves flowing characteristics, increases gloss, and brilliance of film and imparts flexibility to the dried film. These oils have been used in semi-flat paints where the color is of no importance. Blown linseed may be used in the formulation of flat oil paint vehicles.

If the linseed oil is blown to a viscosity $\mathrm{B}$ to $\mathrm{E}$, acid No. 2-6 may be used in printing inks, lithographic varnish, patent leather finishes, and with elastic transparent films of high gloss. They are also used in low grade enamels with "gloss oils."

\section{Bodied Linseed Oil}

The heat bodying, heat polymerization or kettling of oils is probably the oldest and most widely used method for modification of raw or refined oils. Bodied oils, or stand oils as they are called, retain to a large degree the properties of the oil from which they are made, but all bodied oils have certain properties peculiar to the process. The oils bave an acid value which range from 1 to 22 and viscosity of $O$ to $Z_{9}$ or may be bodied in the presence of an inert atmosphere and/or under vacuum. Due to their increased molecular complexity the impermeability of the oil film is greatly increased and penetration into substrate is markedly decreased. These heat bodied oils, 10-15\%, are added to outside house paint vehicles to increase brushability, flow, levelling, hardness, faster drying, better gloss; to improve resistance to water and 
weather; and to decrease corrosion of ferrous supports.

They may be used in paint vehicles to increase wetting characteristics and improve the resistance of the film to water and alkali. For eeonomic reasons a blend of raw linseed oil, heat bodied linseed oil, and mineral spirits in equal volumes makes an excellent vehicle for paints without any sacrifice in consistency. During the interval 1941-1949 the use of heat-bodied oil has increased from $7 \%$ to $67 \%$.

Heat bodied oils are used in water emulsion paints. A heat bodied oil (stand oil) made by the interaction of tung oil and linseed oils during the bodying has superior properties and is used as a high grade exterior paint vehicle. The vacuum bodied oils have an advantage over open kettle polymerized oils in that they have lower acid number and facilitate the removal of decomposition products. The light bodied oils of viscosity $X$ to $Z_{6}$ are used in paints and varnish.

The polymerized oils with acid No. of 7-10, viscosity $Z_{2}-Z_{6}$, on addition of $10-15 \%$ by weight of paints improve the gloss and durability of the dried films.

Polymerized oils produced in hermetically closed kettles with a small outlet under a blanket and current of $\mathrm{CO}_{2}$ to remove decomposition gases, without exposure to air, yield bodied oils the same as open kettles with an acid number of 6 to 18 and viscosity of $\mathrm{Q}$ to $\mathrm{Z}_{4}$. These oils are used to produce over-night drying wood fillers, in which the oil will remain dispersed in the filler, while an ordinary oil would leave the surface with very little oil dispersed in the filler.

Vehicles for non-ferrous metal paints which contain bodied oil in conjunction with treated linseed oil are used to increase the adhesion.

Replacement oil made according to Federal Specification TT-0-371, which is $1 / 3$ heat bodied oil; $1 / 3$ raw or boiled linseed oil and $1 / 3$ naphtha, is an excellent article deserving of special mention as it has placed a scientifically heat bodied oil into the hands of home painters. The replacement oil in some respects is better than vehicles composed from boiled raw, or alkali refined linseed oil. It has more elasticity, is more durable, more water-proof; and less dependent upon the vagaries of raw agricultural crop products. The irregularities in linseed oil from crop to crop are equalized by the heat bodying process. If the raw oil is extended to $75 \%$ of the non-volatile of the blended oils, the product has lost practically all its merit.

\section{Boiled Linseed Oil}

Boiled linseed is produced from raw or alkali refined linseed oil by the incorporation of $0.2 \%$ metals as $\mathrm{Pb}, \mathrm{Mn}, \mathrm{CO}, \mathrm{Zn}$, ete., by means of agitation of soluble, stable metal compounds or by means of a combination of heat and agitation. These oils dry in about one-tenth the time of raw linseed oil and are employed in either interior or exterior paints. They have a viscosity range of $A$ to $B$ (G.H.S.). They may be used in place of raw oil and dryers. The oil is practically free of moisture, and color is darker than the color of the oil from which it was processed. Bodied oil exhibits quick set, controlled flow, good durability, better color retention and water resistance than raw linseed oil in outside house paints. It is used to add to exterior paints as the painter does not need to consider drier addition. If the metallic salts are incorporated by means of heat, the oil may have a high acid number hence it should be used with nonreactive pigments to reduce livering.

Combined with limed rosin it makes an economical interior paint vehicle.

Boiled oils may be had which will dry, in either 12-14 hours or in four hours. The 10-14 hour drying boiled oils are used with slow-drying black pigments. The four-hour drying boiled oils may be used with advantage in hard-to-dry black pigments or railroad and bridge paints.

The boiled oils which have an acid number of 6-8 and a light color may be used for grinding and letting down white and light tint finishes, which are usually brilliant and lustrous films.

\section{Alkali Refined Linseed Oil}

Alkali refining of raw linseed oil removes most of the free fatty acids, break (sugars and mucilagenous material), sterols, color, and other objectionable impurities. This oil is used as a component of low acid vehicles. Retention of color under heat and normal conditions, better initial color, together with its low acid value makes alkali-refined linseed oil a favorite in varnishes, and a source of linseed oil fatty acids or the monoglycerides of linseed oil in the production of alkyds. The viscosity of alkali refined oil is not very different from the raw oil used in its production. The alkali refined linseed oil has little dispersion action on pigments.

This oil is recommended for the formulation of white paints, white enamels, light colored varnishes, alkyd resins of low color, low acid number printing inks, grinding oil for basic pigments and allied products.

\section{Soybean Oil}

Soybean oil is a young rival of linseed oil. It is a good example of the manner in which the paint and varnish industry has seized upon a domestic oil, available in great quantities at a low price, and has adapted it to its use. An examination of the characteristics of refined soybean oil discloses the fact that its films dry slowly and are quite soft, but on the other hand the films have increased flexibility, less checking and less brittleness, better color and color retention than linseed oil films. Tackiness and stickiness of the oil film is most noticeable in reaction pigments, which form slow and soft drying soaps.

Raw soybean oil is of little use in paint films because it sets in more than twice the time required by a linseed oil film. Although the film after it has set is quite durable, it is so soft that it freely collects dirt, supports mildew growth, and soon becomes unsightly. Unmodified soybean oil bodies very slowly in the varnish kettle even at $600^{\circ} \mathrm{F}$. The oil becomes dark and has a high acid number. In spite of the poor showing of raw soybean oil it is of special interest since it contains less than $7 \%$ linolenic acid.

Alkali refined soybean oil is used in house paints without impairing the useful life of the paint film, but its films collect dirt readily and in most ways retain essentially the drying characteristics of raw soybean oil. In general, the direct substitution of soybean oil for linseed oil in a paint retards the drying rate to a degree that is approximately proportional to the amount of oil substituted, but once the film is dry, its durability compares favorably with that of a linseed oil house paint film except for the increased 
dirt collection and increased mildew susceptibility. In paint vehicles small amounts of soybean oil, 15$20 \%$, substituted for linseed oil do not seriously affect the drying time but materially improves the flexibility. With perilla-soybean oil blends large additions of soybean oil are possible. A $15-20 \%$ blend of alkali refined soybean oil and linseed oil is an excellent vehicle to use in grinding colors which will keep indefinitely without skinning or becoming hard. Refined soybean oil does not break below $600^{\circ} \mathrm{F}$.

The different kinds of oils will vary in color and color retention. Soybean oil excels in these characteristics, followed by perilla, linseed, and fish oils.

In the oil conservation type of paint vehicles the replacement of part or all of the bodied linseed oil with bodied soybean oil of equivalent acid number gives results which appear to be as satisfactory as those obtained with the corresponding linseed oils. The higher pigment volume used in these paints and the slower drying are not as apparent, and the higher rate of chalking under normal exposure conditions makes dirt collection much less serious. The tendency to crack increases with increasing body of the soybean oil if the comparison is made at equal volume relations.

Where bodied soybean oil is to be a part of a vehicle, more satisfactory results are obtained by copolymerization with other oils than by blending the bodied oils which have been bodied separately.

Although a blend of soybean and perilla oils in the ratio of 1:2 bodies slowly, the bodied product equals equivalent linseed oil in drying characteristics and may be used in place of a similar grade of linseed oil.

Tung oil bodied with soybean oil yields a bodied oil which is said to be free of the defects of either original oil. Should the soybean oil be prebodied before the co-bodying with tung oil, it has been shown definitely to shorten the drying time of the final bodied oil. Tung or oiticica oil varnish films which tend to become exceptionally brittle on exposure will have this tendeney minimized, and the durability will be improved without hindering other properties of the varnish if some soybean oil is used to replace a portion of either conjugated oil.

Oxidized or blown soybean oil has many favorite uses, among which are printing inks, linoleum, oil cloth, treated fabrics, leather dressing, pigment pastes, and lithographic varnish. The blown soybean oils do not dry out as brittle as the blown linseed oils. The blowing of soybean oils inereases the hydroxy? number, which contributes to its increased solubility in alcohol and a reduction in solubility in mineral spirits, but the blown oil is never completely insoluble in mineral spirits because of saturated fatty acids or their glycerides: The heavier bodied blown oils are used in inexpensive paints and enamels, putty, caulking compounds, and inexpensive plasticizers.

Films of soybean-tung oil blends of a $67: 33$ ratio dry at approximately the same rate as a bodied linseed oil of the same viscosity in paint films, but slightly slower in varnish films. Oils of this blend have practically the same water resistance in films as linseed oil films.

Soybean oil which has been treated by transesterification with pentaerythritol, or with maleic anhydride and styrene to produce a modified soybean oil has been found to have less dirt collection and certain other desirable properties, such as shorter drying time and less yellowing than linseed oil films. The dirt collection and mildew tendency (due to soft films) are almost directly proportional to the unaltered soybean oil remaining in the modified oil. The tendency toward cracking and flaking are greatest in the most highly altered oil. Maleic anhydride-soybean oil has been used successfully in barn and traffic paints.

Some soybean oil alkyds show after-hazing on interior use. This can be reduced by combining part of the soybean fatty acids with pentaerythritol rather than polyhydric alcohol, which contains fewer $\mathrm{OH}$ groups. It requires the production of more complex structures to prevent the non-drying radical migration. Such a migration is manifest in the film by a reduction of gloss. If all the free fatty acids are removed from the finished alkyd, "after-hazing" is reduced to a minimum.

The soybean alkyd is added to lacquer to increase gloss and elasticity in place of castor oil; to asbestos type coatings as a softening agent; to high temperature baking oils and Japans to increase elasticity; to gloss finishes and enamels to improve brushing, wetting, and flow. It is also used in making many alkyd resin solutions which are used in enamels of excellent weather resistance; vehicle for primers, and finishes to be applied by brush, spray, roller coating, or dipping. Other alkyd vehicles uses are architectural, stone house trim, sign paints, marine paints, traffic paints, lithographic coatings, porch and floor paints, spar and overprint varnishes, collapsible tubing coatings, pigment grinding, chill back oil, replacement of bodied oil, and government specifications as $52 \mathrm{R} 13 \mathrm{a}$, types I and II. It may be blended with nitrocellulose lacquers, or acid-catalyzed urea formaldehyde resin to meet the requirements of forced-dry production lines of the automotive or furniture industries.

By the proper selection or balance of pigmentation pure refined soybean oil may be used in paints which dry to a film free from dirt collection and mildew tendencies on outside exposure. After-tack in the soybean oil film may be eliminated almost entirely by the addition of small amounts of calcium oxide, and the durability of the film is improved. The formulation must be such that it will balance the defects: slow drying films and soft drying of refined soybean oil films; and the tendency of bodied soybean oil to crack and flake with the inelastic-hard-dry adhesion of other oils and the proper balance of soap forming pigments.

Soybean oils in the proper form may be blended with linseed, perilla, oiticica, dehydrated eastor oil, fish oil, and tung oil in the manufacture of house paints, varnish, baking Japans, baking finishes for automobile, refrigerator, and various drying synthetic finishes. In most instances alkali-refined oil is preferred.

Soybean oil is used in shingle stains as the nonvolatile portion of the vehicle. In caulking compounds its slow drying properties are valuable.

\section{Castor Oil}

Raw castor oil is not classed as a drying oil or used in protective coatings except as a plasticizer for lacquers, leather dressing dopes, or like formulation.

The dehydration of raw castor oil changes it from a non-drying oil to a drying oil with most of the 
ricinoleic acid converted to a linoleic acid, which includes 20-25\% of conjugated isomeric linoleic acid.

The drying time and water resistance of dehydrated castor oil (DCO) ranks between that of tung oil and linseed oil. It bodies in one-quarter to onethird the time of linseed oil under the same conditions. Films of dehydrated castor oil do not whiten on exposure to moisture like linseed oil films, but they swell under water more than tung oil. The film color is superior to tung or linseed oil films. The dehydrated castor oil films are characterized by nonyellowing, odorlessness, good durability, good wetting, non-livering tendencies, good color, good flow, good adhesion, and little after-tack. The dried film of DCO is more flexible and elastic than tung oil films, and unlike oiticica oil films it does not become brittle on aging and has a durability, considered by some as equal to tung oil. The film of DCO does not dry as hard ultimately as linseed oil or perilla oil films. Properly formulated and cooked dehydrated castor oil vehicle films do not frost or wrinkle or web on exposure to gas fumes in the manner characteristic to tung oil.

The above properties are responsible for dehydrated castor oil becoming a favorite for the production of alkyd resins and varnish in which after-tack is overcome. Its non-yellowing characteristies, either in air drying or baking, assures it broad application in varnish employed in formulation of interior gloss paints and enamels. A 15-20 gallon length DCO varnish makes very good clear automobile enamels.

Dehydrated eastor oil is not a direct substitute for tung oil because of the slow drying and its retention of surface tack for several hours. Hence it is difficult to use in the formulation of rubbing varnishes or baking varnishes or enamels where absence of residual tack is important.

Alkyd resins made with DCO are used in refrigerator finishes because of its paleness and non-yellowing qualities. Some medium oil length DCO alkyds are plasticizers for nitrocellulose lacquers, urea, or melamine resin enamels, polyvinyl chloride and chlorinated rubber coatings, where toughness, adhesion, gloss, and weather resistance are important. Alkyds are also used in white baking enamels on refrigerators and ranges when modified with urea or melamine resins.

Medium oil length DCO alkyds modified with rosin are used in baking enamels for home appliance primers where high humidity, moisture resistance, and adhesion are important. They may also be used in architectural and decorative finishes and in mixing varnish.

Dehydrated castor oil alkyds give better total immersion in salt water than linseed or soybean oils because of their non-yellowing and their impermeability to water, fast drving, and good color retention, which were previously associated only with slow drying alkyds of the sunflower oil type.

Short oil DCO alkyds used with or withont urea or melamine resius are valuable vehicles in enamels for metal furnishings, automotive finishes, engines, pumps, signs, primers and surfacers for metal and plasties, force dry sealers and varnishes.

Alkyd resins made from the distilled fatty acids of DCO surpass the non-yellowing and drying properties of linseed and soybean distilled fatty acid alkyd resin. They are suitable for clear coatings and white enamels to be used in both air and baking finishes. Higher viscosity alkyds may be prepared from medium and long oil DCO alkyd resins than from linseed alkyd resin of similar composition. In short, oil alkyd resins to obtain a high viseosity part of the DCO must be replaced with fatty acids from oils like sunflower or soybean oil. The fatty acids from both oils contribute to the qualities of the short oil alkyd resins; the DCO contributes cooking and drying speed whereas the slow drying oil holds back the polymerization long enough for the acid value to reach a minimum.

Alkyd resins also make excellent under-water primer vehicles in conjunction with $97 \%$ red lead.

Dehydrated castor oil may be gelled and then dispersed in unbodied oil for the manufacture of undercoat and enamel vehicles.

Bodied DCO of low, medium, and high viscosities are being successfully used in paints and enamels. These products dry quicker, have better color retention, greater resistance to water, and possibly greater durability than their linseed oil counterparts.

In varnishes which contain linseed oil, rosin, phenolic resin, or zinc resinate with dehydrated castor oil it has been found to be advantageous to replace up to $40 \%$ of the DCO with perilla, new fish oil products, or tung oil to increase hardness in the vehicle intended for high gloss, eggshell whites, and colored enamels for both air drying and low temperature baking finishes.

Many modified phenolies, especially phenolic modified alkyd resins and polybasic acid phenol resins, are well adapted for cooking with DCO. They cook in a short time at $550^{\circ} \mathrm{F}$. without danger of premature gelation. Where the highest weather resistance and chemical resistance are important in vehicles containing DCO, a $100 \%$ phenolic resin should be employed. If the resin is highly reactive, care must be exercised as excessive bodying may cause gelation in the kettle.

Maleic modified DCO is well adapted for interior enamel vehicles because of its whiteness, color retention, and high gloss, air drying and low baking properties. If the vehicle is too long and is overcooked to obtain the required body, more resin should have been used because there is a tendency to after-body, which may lead to gelation in the case of either pigmented and unpigmented products.

The best baking enamel finishes containing DCO are blends of DCO and some other appropriate oil to meet the baking characteristies required in the vehicles.

\section{Oiticica Oil}

Brazilian oiticica oil is quite similar in many of its characteristies to tung oil. It has been on the market about 20 years, and its possibilities have not been fully explored. The desirable properties of oiticica oil are attributed to the high content of keto-conjugated-licanic acid. Raw oiticica oil dries to an opaque wrinkled film similar to tung oil films. The drying rate is exceeded only by that of tung oil. Large quantities of oiticica oil are consumed by the protective coating industry where it dries hard and more gas-proof than tung oil but has the disadvantage of darkening on heating; on drying its aged films are brittle and less waterproof. For severe exterior uses and high water and chemical resistance the use of 
oiticica oil is limited. Pure oiticica oil films yellow hence color must be considered in formulation.

It gells more slowly than tung oil. Gelled oiticica oil may be dispersed in unbodied oil to use in undercoat and enamel vehicles. It lacks petroleum tolerance.

Oiticica oil is used primarily in varnish. Ten to $40 \%$ of oiticica oil, depending upon the performance required, may be substituted for tung oil in many varnishes. Whenever oiticica oil replaces tung oil in rosin, estergum, cumarone-indene or modified phenolic resin varnishes, the drying time is retarded and the water and ehemical resistance is lowered. If $100 \%$ phenolic resin is cooked with $100 \%$ oiticica oil, the varnish produced has exterior exposure resistance equal to similar tung oil varnishes.

Oiticica oil reacts more rapidly with pure alkyl phenolic resins than tung oil ; is inferior to tung oil for durability in cheap boat varnishes; is used as a component in oleoresinous vehicle to produce crystal, crackle, and wrinkle finishes. Oleoresinous vehicles containing oiticica oil and alkyds are blended to control a definite wrinkle pattern in wrinkle finishes. The oil is employed only to a small degree in white gloss paints, enamels; and flat paints, owing to the greater yellowing than linseed and tung oils. It may be safely used in dark tints.

Oiticica oil forms very stable emulsions when mixed with aqueous solutions. It has been suggested as a plasticizer in waxes and polishes. The brittleness of oiticica oil vehicle films may be reduced by the substitution of linseed or similar oils for part of the oiticica oil.

\section{Tung Oil}

The outstanding drying characteristies of tung oil are for the most part attributed to the fact that the oil has $80-90 \%$ eleostearic acid, which has three unsaturated double bonds in a conjugated system per mole of acid.

The eleostearie acid is considered as the primary reason for the very good waterproofness, rapid drying, hard drying, excellent durability, and ehemical resistance of tung oil films. Tung oil films are highly resistant to saponifying agents. The oil bodies rapidly and has great compatibility with various natural and synthetic resins used in varnish manufacture.

The use of raw tung oil in paints is definitely limited by the fact that it wrinkles on drying and may cause frosting, dull appearance, low elasticity, webbing and checking in films of the customary thickness used in paint films. It may wrinkle in the can. The fact that raw tung oil dries with a "matte" or shrivelled appearance is utilized as a valuable asset in the production of wrinkle or erystalline finishes and also helps to control the pattern in baking wrinkle finishes. Raw tung oil may be used in traffic paints to plastieize, increase adhesion and waterproofness of hard drying butadiene styrene resin vehicles. Raw tung oil is used in the formulation of conerete paints.

Tung oil blended with linseed oil in the ratio of 1 to 3 is a good paint vehicle. Tung oil may be blended with perilla, soybean, and fish oil in preparation of outside house paint vehicles. Blends of tung oil with non-conjugated oils are used in the production of shellac, synthetic, natural resin, and asphaltum varnishes to impregnate paper, fabries, and fiber board. Tung oil is blended with DCO and other oils to control the gas proofing more easily.
Chinese tung oil may be stored for long periods of time without apparent deterioration, but the domestic tung oil must be used within a comparatively short time to avoid storage losses.

The rapid bodying of tung oil is controlled in the varnish kettle by the addition of non-conjugated oil as linseed, soybean, fish oil, and many other nonconjugated oils. Tung oil which is heated to $550^{\circ} \mathrm{F}$ for 5-8 minutes is gas proofed and also has several other desirable properties, such as increased acid and alkali resistance, which offset the undesirable characteristics of raw tung oil in many of its uses.

Most of the tung is preferably modified by conversion into stand oil or by incorporation with resins into varnish or blending with non-conjugated vegetable oils such as linseed, soybean, fish, sunflower, and safflower oil. Stand oils in which tung oil is combined with linseed, perilla, or similar oils yield products to provide excellent flow, good gloss, rapid dry, and excellent water resistance are used in high gloss paints and controlled penetration, one-coat paints.

Tung oil makes possible the preparation of spar varnish from estergum, phenolic resin, hardened rosin. Oxidized tung oil varnish films have high insulation properties. Alkyd resins containing tung oil are used in finishes which are air dried and must show characteristics of toughness and hardness. Can and wire enamels are made from 15-30 gallon tung oil varnishes which contain Congo or pure phenolic resins. Beer cans may be covered with a varnish composed of tung oil-rosin maleic glycerin esters. The liquefied gel of tung oil is unsuitable for good varnishes but may be used in eheap varnishes.

\section{Perilla Oil}

Perilla oil has the highest iodine number of all the raw non-conjugated drying oils. It has excellent drying properties, which lie between those of tung and linseed oils. It has high surface tension and creeps when spread in thin films. If heated to $500^{\circ} \mathrm{F}$ for a short time, the surface tension and yellowing tendencies are reduced. The oil dries to a brilliant, hard, tough, and fairly waterproof film. 'The bodying rate of perilla oil is almost twice that of linseed oil but has a greater tendency to yellow, especially on baking. It has a lower flash point than most oils. The odor is pleasant.

Perilla oil is used to fortify linseed oil, soybean oil, and semi-drying oils and to make blended oils which simulate linseed oil in drying characteristics. A nonbreak perilla oil is used to substitute for linseed oil or tung oil in varnishes which require less drier and have good color retention. Blown perilla oil is noted for its rapid drying, the hardness of its film, and resistance to weathering.

It has been used to produce alkyd resins and is considered by some a favorite in varnish making. Perilla oil may be gelled, and then the gelled oil may be dispersed in unbodied oil for use in the manufacture of undercoat and enamel vehicles. It is used in the production of printing inks. It has been styrenated to form modified oils. The oil is being used less today because of cost and supply factor.

\section{Fish Oils}

The principal marine oils which are of special interest to the paint manufacturers are menhaden, salmon, and sardine oil. Unlike other paint oils, these 
oils have some tetra- and penta-functional acid chains which are extremely fast drying. These oils have excellent adhesion, elasticity, and durability. They are not as water-resistant as tung oil, but because of their elasticity and adhesion the water resistance is better than commonly used oils. Their wetting properties for equal body are superior to those of vegetable oils. Refined fish oils have relatively good non-yellowing characteristies and do not blister as much on baking as many vegetable oils. The refined oil has a large part of the saturated fatty acids removed. The iodine number of the individual unsaturated fatty acid radicals in fish oil range from 75 to 360 , the average iodine number is 180-190. Refined fish oil does not break.

Processing above $590^{\circ} \mathrm{F}$ causes a rapid increase in acidity. It has been found a temperature of $560^{\circ} \mathrm{F}$ is commonly used to process fish oil in saturating the highly unsaturated components without affecting materially the lower unsaturates.

Sardine oil which has been bodied to 9 poise at $535^{\circ} \mathrm{F}$ and then further bodied to a viscosity of 98 to 148 poise by air blowing, when cut back with thinner, retains the improved flow, brushing, and levelling qualities of oxidized oils.

Fish oils are generally used in oil blends with perilla and linseed oil. The fish oil films are slow drying, have after-tack especially at high humidities, poor strength, and a characteristic odor. It is used by some manufacturers as part of a cheap paint vehicle. Raw fish oils have been used in high temperature baking Japans, barn paints, house paint, fence paint, railroad box car paint, anti-rust paint, aluminum paint, industrial and maintenance paints, struetural paint, enamel vehicles, semi-gloss paints, fattening lacquers, and primers for wood surfaces. Fish oil is used in short, medium, and long oil varnishes of the phenolic type, synthetic varnish type, and all oil varnish types. The oil may be added directly in first cook, check, or eut back.

Fish oil and linseed oil blends used in house paint vehicles show less dirt pickup than pure fish oil vehicles. Fish oil may be blended with tung, linseed, oiticica, perilla, or soybean oils. Care must be exercised to formulate correctly to reduce after-tack and undesirable soft film properties. Fish-tung oil blends are used in smoke stack paints that will withstand heat and light.

Fish oils are used in eaulking compounds because of surface dry and flexible interior, and freedom from bleeding, staining, shrinkage, and sagging. It is also used in roof paints and rough lumber paints.

In short oil varnishes the addition of fish oil improves the elasticity and color, lessens skinning, and increases compatibility with pigments. The addition of fish oil to medium oil length cooks of varnish helps to insure gas-proofing and helps in the dispersion of the polymers, thus redueing skinning and gel tendencies and decreasing the reactivity with basic pigments.

The long oil varnishes, such as mixing varnishes, spar varnishes, finishing varnishes, railroad varnishes, enamel varnish vehicles, and industrial paints, all have good waterproofness and good exterior properties. Industrial liquids and mixing vehicles incorporate refined or bodied fish oil to reduce cost and meet certain specific industrial requirements as railroad paint and pipe coating paints. Lightly kettled fish oils are used in stock pastes and colors in oil and paste paints because of their non-skinning, color retention, color, absence of after-yellowing, and clarity of tints.

\section{Tall Oil}

Tall oil, a by-product of the sulfate pump industry, is composed of $45-50 \%$ fatty acids (principally oleic and linoleic acid), $42-48 \%$ rosin acids, and $6-9 \%$ sterols. The rapid increased consumption indicates it is finding a place in modern industrial protective coating manufacture. The refined tall oil esterified with glycerol or pentaerythritol produced a product which is roughly equivalent to a 12-gallon linseedestergum varnish.

Although its use in paints has not become general and it is considered a low cost diluent, yet tall oil is a monument to the ingenuity, imagination, perseverance, and ability of modern science to convert a byproduct into a valuable adjunct of modern protective coatings. Tall oil films dry in a reasonable time to a hard tack, free film in paints. At present its use is most in the more inexpensive paints. The steadily increasing supply, at the present time, is being absorbed by the linoleum, paint, varnish, soap, lubricant, and allied industries.

It is esterified with polyhydric alcohols. The products are used in rust-proofing, water-proofing, coatings for casks, floors, furniture, and similar applications. The esterified tall oil products are darker than the corresponding linseed oil products, slower drying, and less elastic. The same may be said of similar tung oil and oiticica oil products. The weather resistance of tall oil is improved on blending with tung oil, estergum, copal ester, and some synthetic resins. It makes a good component in short oil varnish.

\section{Other Oils}

There are a large number of other oils which have been investigated. Some have been used in a limited amount in special formulations. Most of these oils have one or more defects, when compared with the major drying oils, the chief of which is unavailability. The more important of these oils are Po-yoak oil, poppy seed oil, chia oil, stillingia oil, sunflower oil, safflower oil, hempseed oil, walnut oil, corn oil, rape seed oil, sesame oil, cashew nutshell oil (liquid), bonito oil, lumbang oil, nigerseed oil, grapeseed oil, milkweed seed oil, and many others. The fact that these oils may be more or less scarce at this time has not hindered the continued investigation of their properties. Many of these oils, like safflower oil, will in time be recognized for some particular properties which will stimulate their production.

Hempseed oil dries more slowly than linseed oil, but there are no yellowing tendencies on storage. The oil film in outside house paint has low water absorption and hence low film emulsification tendeneies. Its durability is as good as linseed oil in outside house paint. It dries in a way similar to poppy seed oil but more slowly than linseed oil. Boiled four hours, the drying time is the same as linseed oil similarly treated. Because of its low staining power it makes an excellent grinding vehicle for colors in oil. It has higher dispersing power than linseed oil. The faint bluish color of the oil is an asset in grinding white pigments. It may be used to cook Congo resin varnishes and alkyd resins. It is blended with linseed or perilla oil for outside house paint vehieles. 
Sunflower oil, a semi-drying oil, yellows less than linseed oil, but it dries softer and more slowly. Its use in varnish oil at present is limited by its darkening characteristics. It dries more slowly than soybean oil. It may be blended with high iodine number linseed oil and perilla oil for use in outside house paints. It makes good alkyd resin solutions and can be used in varnish in conjunction with drying oils.

Raw chia oil drys slowly and because of its high surface tension collects in drops. The tendency to form drops may be eliminated if it is heated to $410^{\circ} \mathrm{F}$ for 15 minutes. In drying it is superior to linseed oil treated with the same amount of drier. There is little change in color after heat bodying hence it is used in light colored varnishes or alkyd resin. If the supply is developed, it may be used in exterior paints and enamels.

The poppyseed oil dates from the time of Dioscorides and was used before linseed oil was known. Poppyseed oil might be substituted for soybean oil if it were more plentiful. It has been used since the 17th century by Dutch artists who appreciated its slow drying properties, but painters preferred linseed oil. The oil tends to retain its color with age and requires more drier than linseed oil. The oil film tends to show eracking if the pigments are too finely ground. Poppyseed oil films dry softer and more slowly than linseed oil.

Oxidized poppyseed oil is more soluble in solvents than linseed oil. Poppyseed oil does not have the keeping properties of linseed oil. Glycerides of poppyseed oil are easily hydrolyzed, and the acid value of the oil increases on exposure. Thickened poppyseed oil has a reduced drying time and the films show little tendency to crack. Because of supply it cannot compete in house paint with linseed or similar oils.

Cottonseed oil without modification has little use in paints. It dries more slowly than soybean oil and remains soft for long periods of time; the films pick up dirt. Its use in the manufacture of alkyds resin has proven to be of considerable value. It can be used as a plasticizer, also in varnish with other oils.

Corn oil is a member of the semi-drying oil class. Its drying characteristies lie between soybean oil and cotton seed oil. The refined oil may be water-white in color. It has been used in blends with tung oil and linseed oil up to $10 \%$ of the total oil content for a vehicle in ready-mixed paints to help prevent hard settling. Corn oil in some localities is used in oil blends to produce vehicles for barn and outside house paints, where the corn oil content should be kept low to prevent soft drying. Blended with linseed oil it has found favor as a non-skinning, grinding vehicle for paste colors. The oil does not gelatinize on heating. It would be used more in alkyd resins if the supply could be increased. Corn oil is used in the manufacture of linoleum, oil cloth, and varnish. Stand oil prepared from corn oil is a component of good drying enamels, which do not yellow as much as similar enamels that contain linseed or tung oil.

Ravison oil of good quality may be difficult to procure since its properties are greatly influenced by the growing environment and eultivation techniques. The oil of commerce varies from yellow to a dark brown. The high percentage of isolated double bond fatty acids, $70-80 \%$, has limited the commercial application of the raw and blown oil to the roll of plasticizers in nitrocellulose lacquers or similar types of application.
The drying characteristics are regarded as being between that of non-drying and semi-drying elass of oils. It has been used in alkyd resins.

Sesame oil is a member of the semi-drying oil class. The refined oil may be blended with perilla oil or linseed oil to produce outside house paint vehicles, providing not too much sesame oil is used to render the dried film soft. It has been used in alkyd resins, linoleum, and soap.

Cashew nutshell oil is a by-product of the cashew nut industry. It is not a triglyceride as are the other natural oils discussed but is considered to be a modified phenolic liquid resin. The reduced cashew nutshell oil is used in the formulation of cement paints, industrial paints, marine paints, and chemical resistant paints. It makes a good plasticizing oil. Blends with vegetable oils are used in floor varnish where it is reported to improve toughness, flexibility, and waterproofness to the dried film. It is used as a solvent for pitches, asphalts, and resins. It polymerizes when heated and has been used to reduce the baking temperature of baking enamels. It is used in formulations with many synthetic resins, natural resins, aldehydes, alkaline earth oxides, and hydroxides, polyhydric alcohols, alcohols, and some amines to form valuable products. Sulfuric acid causes cashew nutshell oil to polymerize to a resin of exceptional toughness and resistance to wear, abrasion, and high temperature.

Stillingia oil resembles linseed oil in its chemical and physical properties. It should be classed as a dry. ing oil, but since the supply is scarce on account of lack of interest in the cultivation of the stillingia tree and the difficulty in harvesting and separation of the oil from other undesirable fatty and waxy products, it is discussed in this section of the paper. It can be substituted for linseed oil in paints, varnishes, and alkyd resins.

Walnut oil is similar to hempseed oil in many of its properties and dries like soybean oil. Hot pressed walnut oil has been used in paints and artists' colors from early times. It reacts with styrene in the presence of a catalyst to produce excellent styrenated oils. It has been used in varnish and alkyd resin. Its use would become general if an adequate supply were available at a price less than that of linseed oil.

Safflower oil has been used as an edible oil like many of the other semi-drying oils for centuries. Not until recent years has it been considered seriously as a protective eoating vehicle in the United States. The major portion of the fatty acids found in safflower oil is linoleic acid; there is less than $1 \%$ of linolenic. The raw oil dries better than soybean oil. There is no after-yellowing in the dried film, even under extreme exposure conditions. The oil is easily bleached to a water-white color which is equivalent in color to highly refined corn oil. It has been stored eight years without any noticeable effect on its clarity, color, luster, or acidity. The oil solidifies at $8^{\circ} \mathrm{F}$ but remains clear.

Boiled oil and stand oil prepared from safflower oil show better properties than the raw oil. It is less durable than linseed oil and may be used in place of poppyseed oil in artists' colors. The oil does not break when heated to $600^{\circ} \mathrm{F}$ or discolor. Heated to $600^{\circ} \mathrm{F}$ for 2.5 hours, the oil suddenly gels, but the gel, unlike tung oil gel, is soluble in turpentine and 
many other organic solvents. The acid number is lower than most vegetable oils similarly treated:

Safflower oil is used successfully in house paints, especially if blended with tung oil, linseed oil, or perilla oil. It has been used to make styrenated oils. Boiled oil and bodied oils are non-breakable and are used to produce varnishes with synthetic and natural resins. Raw or bodied safflower oil makes good alkyd resins. As production increases, safflower oil will become more popular among protective coating formulators.

\section{Future of Synthetic Oils}

Now that we have considered the use of the more important vegetable oils in paint, just a word in regard to the future. There are numerous patents and applications for patents directed toward the production of synthesized oils from non-fatty acid raw materials. 'To date in spite of the tremendous amount of time, expense, and talent being directed toward the development of a suitable replacement material for the fatty acids in drying oils, the ideal solution is not in sight. If and when the new process is developed, the vegetable oil industry will face stiff competition, provided it is plentiful, cheap, free from seasonal fluctuation, and independent of climatic variations. At the present stage of synthetic oil development the score is in favor of the natural oils as slow-drying. yellowing, and severe checking on aging are the hurdles which continue to present problems that must be surmounted.

There are many claimants for the vegetable oil "oscar" in protective coatings. Styrenated petroleum products of butadiene, acryleates, vinyls, acetylates, rubber polymers, halogenated aliphatic and aromatic organic compounds are among those which have been suggested and introduced to the market but have found only limited application in specialties. Vegetable oil scientists are not asleep. They know they have a valuable and abundant raw material supply and are releasing methods for temperature treatment of semi-drying oils like grapeseed, rapeseed, and tomatoseed oils to convert them into materials which have drying properties similar to linseed oil. Svnthetic tung oil produced from fish oil and synthetic linseed oil processed from coal oils are realities. The continued development of the synthetic oils will be watched and will assume increasing importance as commercial competitors of vegetable oils.

Any changes from vegetable oils to synthetic oils will be slow as manufacturers are loath to change from an established product, even though the new product may be superior, until it has been established beyond any reasonable doubt that they can depend upon an adequate, stable, available, and steady-priced material for their production schedule.

\section{REFERENCES}

I. Books

1. Bailey, A. E., "Industrial Fats and Waxes," Interscience Pubishers Inc, (1945)

2. Bartell, F. E., Laboratory Manual, "Colloid Chemistry," Edwards Press, Ann Arbor, Mich. (1947).
3. Bearn, J. G.; "The Chemistry of Paints, Pigments and Varnishes," Ernst Benn Litd" (1923)

4. Blom, A. V., "Organic Coatings in Theory and Practice," Elsevier Publishing Company, New York (1949).

5. Chatfield, H. W., "Varnish Constituents," Interscience Publishers nc., New York (1944).

6. Heston, Noel, "Outhines of Paint Technology," 3rd Edition Charles Griffin and Company, Ltd., London (1947).

7. Hilditch, T. P., "The Industrial Chemistry of Oils, Fats, and Waxes," John Wiley and Sons Ine., New York (1941).

8. Huff, Ralph H., Scientific Methods of Varnish Manufacture American Paint Journal Company, St. Lou is, Mo. (1946).

9. Jamieson, G. S., "Vegetable Fats and Oils," 2nd Edition, Reinhold Publishing Corporation, New York (1944).

10. Joachin, Benjamin, "Applied Paint and Varnish Chemistry," Vol. I, Pigments and Oils; American Paint Journal Company, St. Louis, Mo. (1934).

11. Kirschenbauer, H. G., "Fats and Oils," Reinhold Publishing Corporation, New York (1944)

12. Lewkowitsch, J., "Chemical Technology of Oils, Fats, and Waxes," Vol. II and III, Macmillan and Company, London (1923).

13. Martin, R., "Lacquer and Synthetic Enamel Finishes," Van Nostrand Company, New York (1946)

14. Mattiello, J.' J., "Protective and Decorative Coatings," Vol. I and IIr., John Wiley and Sons Inc., New York (1947).

15. Morrell, R. S., and Wood, H. R., Chemistry of Drying Oils, Van Nostrand and Company, New York (1925).

16. U. S. Department of Agriculture, "The Fats and Oils Situation," uly 1947 and $(1949)$.

17. Von Fischer, Carl, "Paint and Varnish Technology," Reinhold ublishing Corporation, (1949).

18. Wampler, R. H., "Modern Organic Finishes," Chemical Pub lishing Company Ine, Brooklyn (1946).

II. TECHNICAL AND TRADE JOURNALS

1. Beavers, E. M., "Alkyd Resin Technology," Am. Paint Jonrnal, 2. Bradley, T. F., and Richardson, D., Ind. Eng. Chem., 34, 237 (1942).

3. Bolley, D. S., Ind. Eng. Chem., 41, 287 (1949)

4. Burrell, H., Official Digest, F. of P. \& V. Prod. Clubs, p. 326, April 1948 .

5. Chatfield, H. W. Paint $X I X$ (4), 115 (1949).

6. Christensen, L. M., Am. Paint Journal, 34, (20), 54 (1950)

7. Cosgrove, C., and "Earhart, K. A., Ind. \& Eng. Chem., 41, 1942 (1949).

8. Eisenschiml, O,, Am. Paint Journal, 32, 72 (1947).

9. Falkenburg, L., B., et al, Ind. Eng. Chem., 38,1002 (1946).

10. Greaves, J. H., Paint, XIX, III (1949).

11. Hafeli, J. M., By Gum, Aug.-Sept., p. 7 (1948).

12. Hardy, E. Paint $X I X, 32$ (1949).

13. Hilditch, D. Paint, XIX, 126 (1949).

14. Hovey, A., Official Digest, p. 697 (1949).

16. Kienle, R. H., Official Digest of F. of P. \& V. Prod. Clubs, 11,

January (1950).

17. Konen, J. C., Official Digest, F. of P.\& V. Clabs, p. 647 (1948).

18. Larson, L. P., and Calbeck, J. H., Paint Oil and Chem. Rev.

$112,25(1949)$

19. Lanson, H. J., et al., Ind. Eng. Chem., 37, 179 (1945)

20. Lewis, A. J., Paint Ind. Magazine, 64, 198 (1949). Clubs, Sept.

1949

22. Lovern, J. A,, Paint Ind, Mag., Aug., p. 224 (1949)

23. Mann, L. C., Am. Paint Journal 33,66 (1948)

24. Menaker, A. J., Paint Oil \& Chem. Rev., 113, 36 (1950).

26. Northwestern diub, Official Digest, $F$. of $P . \&$ V. Prod. Clubs,

p. 298 , p. 838 ( 949 )

27. Priest, G. W., and V. Mikusch, J. D., Ind. Eng. Chem., 32, 1314 1940)

28. Radlove. S. B., et al., Catalytic Conjugation of Linseed and Soybean Oils, Northern Regional Research Laboratory, U. S. D. A., Peoria,

Ill., October 1945.

29. Rauber, E. I., Nat. Paint Bull., 10, 3, 5 (1946)

30. Razzano, D. D., Am. Paint Journal, $32,29,1948$

31. Reizenstein, L. J., Drugs, Oil, and Paints, 53, 212 (1938).

32. Reutenauer and Sisley, O. A. 42,8492 (1948).

33. Rice, H. L., Paint and Varnish Prod 12 (Feb. 1950)

34. Rice, $H$ L Paint and Varnish Prod. 8 (Nov 1949).

35. Schwitzer, M. K., Paint Mft. 18, p, 159 and 168 (1948)

36. Schwarcman, A., Official Digest, F. of P. \& V. Prod. Clubs, 325, (Apr. 1948)

37. Stieg, F. B., Official Digest, F, of P. V. Clubs, p. 94 (Feb.

38. Scofeld, F., Official Digest F. of P. \& V. Prod. Clubs, p. 438 (1948).

39. Sutheim, G. M., Chem. Industries, 62, 65, and 241 (1948).

40. Terrill, R. L., and Mitchell, F., Am. Paint Journal, 32, 94 (1947)

41. Touchin, H. R., Paint Mfg., 16, 186 (1946)

42. Wochholtz, W. A., Official Digest, F. of P. \& V. Prod, Clubs, p. 446, (June 1948).

\section{Misceluaneous}

Nat. Paint, Varnish and Laequer Association, Scientific Section Cireulars, Nos: $571,582,622,651,654,656,662,669,672,673$. $697,721,725$. 